\title{
Atrial fibrillation increases inpatient and 4-year all-cause mortality in critically ill patients with liver cirrhosis
}

\author{
Yanting Luo ${ }^{1 \#}$, Bingyuan $\mathrm{Wu}^{1 \#}$, Yuankai $\mathrm{Wu}^{2}$, Long Peng ${ }^{1}$, Zexiong $\mathrm{Li}^{1}$, Jieming $\mathrm{Zhu}^{1}$, Zhongzhen $\mathrm{Su}^{3}$, \\ Jinlai Liu ${ }^{1}$, Suhua $\mathrm{Li}^{1}$, Yutian Chong ${ }^{2}$ \\ ${ }^{1}$ Department of Cardiovascular Medicine, Third Affiliated Hospital, Sun Yat-sen University, Guangzhou, China; ${ }^{2}$ Department of Infectious Diseases, \\ Third Affiliated Hospital, Sun Yat-sen University, Guangzhou, China; ${ }^{3}$ Department of Ultrasound, Fifth Affiliated Hospital, Sun Yat-sen University, \\ Zhuhai, China \\ Contributions: (I) Conception and design: Y Wu, L Peng, Z Li; (II) Administrative support: None; (III) Provision of study materials or patients: \\ None; (IV) Collection and assembly of data: Y Luo, B Wu, Y Wu, L Peng, Z Li; (V) Data analysis and interpretation: Y Luo, B Wu; (VI) Manuscript \\ writing: All authors; (VII) Final approval of manuscript: All authors. \\ "These authors contributed equally to this work. \\ Correspondence to: Yutian Chong. Department of Infectious Diseases, Third Affiliated Hospital, Sun Yat-sen University, Tianhe Road No. 600, \\ Guangzhou 510630, China. Email: chongyt@mail.sysu.cn; Suhua Li. Department of Cardiovascular Medicine, Third Affiliated Hospital, Sun Yat-sen \\ University, Tianhe Road No. 600, Guangzhou 510630, China. Email: lisuhua3@mail.sysu.edu.cn.
}

Background: The association between atrial fibrillation (AF) and cirrhosis is unclear. Therefore, the aim of the present study was to determine the association between AF and short-term and 4-year mortality in critically ill patients with cirrhosis using a large database.

Methods: The Medical Information Mart for Intensive Care III (MIMIC III) database was used to identify patients with cirrhosis hospitalized in an intensive care unit from 2001 to 2012. Demographic and clinical data were extracted from the database. Clinical data and demographic information were collected for each patient in our study. Kaplan-Meier analysis and multivariate Cox regression models were performed to examine the relation between atrial fibrillation and in-hospital and 4-year all-cause mortality.

Results: A total of 1,481 patients (mean age: 58 years, $68 \%$ male) with liver cirrhosis were included in the analysis, and the prevalence of $\mathrm{AF}$ was $14.18 \%$. The inpatient all-cause mortality rate was $26.6 \%$, and patients who died in hospital had a significantly higher rate of $\mathrm{AF}(21.57 \%$ vs. $11.50 \%, \mathrm{P}<0.001)$. Multivariate Cox regression analysis indicated that AF was significantly associated with inpatient all-cause mortality [hazard ratio (HR): 1.52, 95\% confidence interval (CI): 1.19-1.95, $\mathrm{P}<0.001$ ], and 4-year all-cause mortality (HR: 1.55, 95\% CI: 1.12-2.13, P=0.008). Kaplan-Meier survival analysis showed that patients with AF had a significantly higher inpatient and 4-year all-cause mortality.

Conclusions: Critically ill patients with liver cirrhosis have a high rate of AF, and the presence of AF is an independent risk factor for inpatient and 4-year all-cause mortality.

Keywords: Atrial fibrillation (AF); liver cirrhosis; critically ill patients; mortality; anticoagulation

Submitted May 25, 2021. Accepted for publication Jul 22, 2021.

doi: $10.21037 / \mathrm{atm}-21-3111$

View this article at: https://dx.doi.org/10.21037/atm-21-3111

\section{Introduction}

Liver cirrhosis is a chronic, progressive disease that is primarily caused by excessive alcohol consumption and viral hepatitis, and can eventually lead to complete liver failure (1). In the decompensated stage of liver cirrhosis, hemodynamic disturbances, activation of the reninangiotensin-aldosterone system, increased sympathetic nervous system activity, and increased secretion of inflammatory cytokines and cardiotoxic substances can result in cardiovascular dysfunctions (2,3). Cirrhotic 
cardiomyopathy and atrial fibrillation (AF) are also common in liver cirrhosis, as well as gastrointestinal diseases (4).

$\mathrm{AF}$ is the most common sustained cardiac arrhythmia in adults and is associated with significant morbidity and mortality $(5,6)$. It is also associated with various gastrointestinal and liver diseases, such as non-alcoholic fatty liver disease and liver fibrosis $(7,8)$. Liver cirrhosis has been found to increase the risk of cardiac arrhythmias (9), and a number of studies have shown that cirrhosis is a risk factor for $\mathrm{AF}(10,11)$. However, the impact of AF on mortality in patients with liver cirrhosis is unclear (10,12-14).

Therefore, the aim of the present study was to determine the association between $\mathrm{AF}$ and short-term and 4-year mortality in critically ill patients with cirrhosis using the Medical Information Mart for Intensive Care III (MIMICIII) database, which contains information on intensive care unit (ICU) admissions.

We present the following article in accordance with the STROBE reporting checklist (available at https://dx.doi. org/10.21037/atm-21-3111).

\section{Methods}

\section{MIMICIII database}

Data used in the present study were extracted from the MIMICIII database $(15,16)$. The database was developed by researchers from the Laboratory for Computational Physiology at Massachusetts Institute of Technology (MIT; Cambridge, MA, USA) and the Department of Medicine at Beth Israel Deaconess Medical Center (BIDMC; Boston, MA, USA). The database contains detailed information on patients who have been treated in the ICU, including patient age and sex, clinical and laboratory data, radiography reports, treatments, discharge summaries, and follow-up information. It also contains diagnoses based on International Classification of Diseases, 9th Revision (ICD-9) codes. At the time of the present study, the database contained information of 58,976 patients treated in the ICU at BIDMC from 2001 to $2012(15,16)$. The CareVue and MetaVision systems are the 2 systems within the database that follow-up data can be extracted from, and both were used in the present study. The database is accessible to researchers who have completed a 'protecting human subjects' training.

The Institutional Review Boards of the BIDMC and MIT approved the use of the MIMICIII database for the present study (certification number: 1564657). Because of the retrospective nature of the study, the requirement of informed patient consent was waived. Data extraction was performed using PostgreSQL tools V.1.12.3. The study was conducted in accordance with the Declaration of Helsinki (as revised in 2013).

\section{Study population and variables}

Patients treated in the ICU between 2001 and 2012 who met all of the following criteria were included in the analysis: (I) age $\geq 18$ years; (II) primary diagnosis of liver cirrhosis (ICD-9 codes 5712, 5715, 5716); and (III) length of ICU stay $\geq 24$ hours. Data extraction was performed using PostgreSQL tools V.1.12.3, and the following data were extracted in the first 24 hours of ICU admission: (I) ICU type (e.g., cardiac ICU, medical ICU); (II) demographic information, including age, sex, ethnicity, weight, and height; (III) clinical data, including heart rate, blood pressure, AF, and disease severity, as assessed by the model for end-stage liver disease (MELD) score and the sequential organ failure assessment (SOFA) score $(17,18)$; (IV) laboratory data, including hemoglobin, white blood cell (WBC) count, platelet count, international normalized ratio (INR), red cell volume distribution width $(\mathrm{RDW})$, and serum albumin level; and (V) prescription data, including the prescription of anticoagulation (AC) and the type of AC.

Patient history of cardiovascular and other comorbidities that can be related to liver cirrhosis, including hypertension, heart failure, coronary artery disease, $\mathrm{AF}$, diabetes, chronic kidney disease (CKD), cerebral embolism, cerebral hemorrhage, and gastrointestinal bleeding, was also extracted.

Study participants had to meet the inclusion criteria to reduce information bias. Multivariate regression analysis was used to reduce repetition bias.

\section{Study outcomes}

The outcomes of the present study were inpatient and 4-year all-cause mortality. For patients who survived and were discharged, the date of death was obtained from US Social Security Death Index records.

\section{Statistical analysis}

Continuous variables were presented as mean \pm standard deviation or median [interquartile range (IQR)], and categorical variables were presented as count (percentage). 


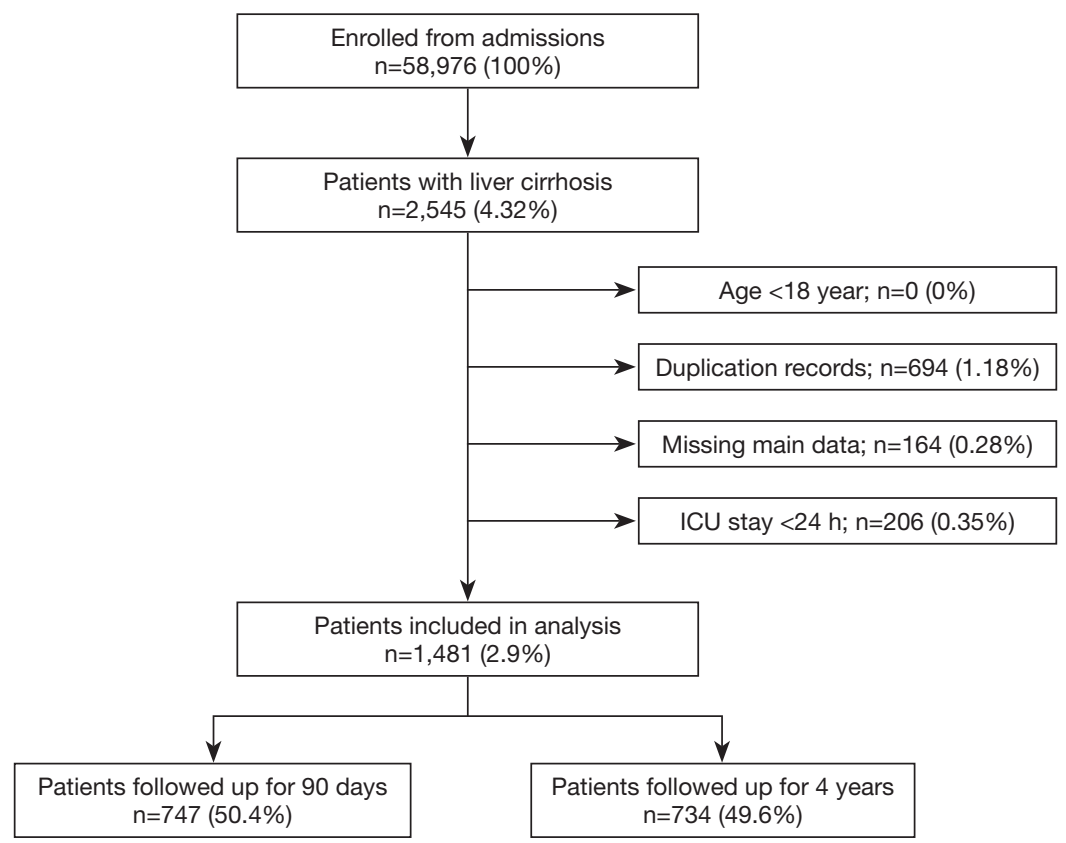

Figure 1 Flow diagram of patient inclusion. ICU, intensive care unit.

Student's independent $t$-test or Wilcoxon rank sum test was used to compare the means of continuous variables, depending on the assumption of normality. Categorical variables were compared using $\chi^{2}$-test.

Of all the variables in the dataset, the missing value ratio of each variable was less than $10 \%$. The missing values were imputed by expectation-maximization method using SPSS version 26 (IBM, Armonk, NY, USA).

The Kaplan-Meier method was used to provide survival estimates and to evaluate the association between $\mathrm{AF}$ and inpatient and 4-year mortality. Association between AC administration or not in cirrhosis patient with AF and inpatient and 4-year mortality was also evaluated. Landmark analysis was used to assess all-cause mortality occurring before and after 2.5 years of follow-up (19). Differences were examined by log-rank test. Univariate and multivariate Cox proportional hazard regression models were used to investigate factors associated with inpatient and 4-year mortality. Independent variables examined included age, sex, body mass index, smoking, coronary heart disease, diabetes, hypertension, heart failure, CKD, SOFA score, MELD score, platelet count, albumin level, hemoglobin level, WBC count, RDW, INR, and AF. Variables with $\mathrm{P}<0.05$ in the univariate analysis were entered into the multivariate model. Variables that were found to be significant $(\mathrm{P}<0.05)$ in both the univariate and multivariate analyses were considered to be factors associated with mortality. With the exception of data consolidation which were done using Stata version 12.0 software, all of the analyses were done using SPSS version 26. In all of the analyses, a 2-tailed value of $\mathrm{P}<0.05$ was considered to indicate statistical significance.

\section{Results}

\section{Study population characteristics}

Of the 58,976 ICU admissions in the MIMICIII database between 2001 and 2012, a total of 2,545 patients were diagnosed with liver cirrhosis, of which 694 were excluded because they were duplications. All patients were $\geq 18$ years of age; however, 164 records were excluded because of missing data of interest and 206 were excluded because the ICU length of stay was $<24$ hours. Therefore, $1,481 \mathrm{ICU}$ admissions were included in the analysis. A flow diagram of patient inclusion is shown in Figure 1. A total of 734 patients with data in the CareVue system were followed up for 4 years, and 747 patients with data in the MetaVision system were followed up for 90 days after discharge.

The mean patient age was $58.53 \pm 10.99$ years, and 1,002 patients were male $(67.66 \%)$. The leading defined cause of cirrhosis was alcohol abuse ( $\mathrm{n}=713,48.14 \%)$; however, in approximately half of patients $(n=739,49.9 \%)$ the cause was listed as "unclassified". The overall prevalence of AF 
in ICU patients with liver cirrhosis was $14.18 \%$. A total of 394 patients died during hospitalization; therefore, the inpatient all-cause mortality rate was $26.6 \%$. Patients who died in hospital (non-survivors) had significantly higher rates of $\mathrm{AF}(21.57 \%$ vs. $11.50 \%, \mathrm{P}<0.001)$ and heart failure $(20.30 \%$ vs. $14.44 \%, \mathrm{P}=0.007)$, and a lower rate of hypertension $(22.84 \%$ vs. $28.70, \mathrm{P}=0.025)$ than survivors.

Characteristics of survivors and non-survivors are shown in Table 1. Non-survivors had significantly higher MELD scores [10.74 (IQR: 7.13-17.08) vs. 5.29 (IQR: 3.95-7.96), $\mathrm{P}<0.001$ ] and SOFA scores [9 (IQR: 7-13) vs. 6 (IQR: $4-8), \mathrm{P}<0.001]$ than survivors. Non-survivors also had a significantly higher WBC count [11.10 (IQR: 7.40-16.73) vs. 9.40 (IQR: 5.70-12.20), $\mathrm{P}<0.001$ ], RDW [19.60 (IQR: 17.60-22.10) vs. 18 (IQR: 16.30-19.67), $\mathrm{P}<0.001$ ], and INR [1.99 (IQR: $1.60-2.60)$ vs. 1.60 (IQR: $1.40-1.82$ ), $\mathrm{P}<0.001]$ than survivors. Non-survivors also had significantly lower mean arterial pressure, albumin level, hemoglobin level, and platelet count than survivors (all $\mathrm{P}<0.05$ ) (Table 1).

\section{Comparison based on AF quartiles}

Unadjusted outcomes of patients with and without AF are summarized in Table 2. The cause of cirrhosis was similar between the 2 groups. Without adjusting for covariates, the inpatient all-cause mortality rate was significantly higher for patients with $\mathrm{AF}$ than those without $\mathrm{AF}$ (40.48\% vs. $24.31 \%, \mathrm{P}<0.001)$. ICU length of stay and hospital length of stay were significantly longer, and MELD score was significantly higher in patients with $\mathrm{AF}$ (all $\mathrm{P}<0.05)$; SOFA score was similar between the groups.

\section{Association between AF and study outcomes}

Univariate and multivariate Cox proportional hazard regression models were used to evaluate factors, including $\mathrm{AF}$, with inpatient and 4-year all-cause mortality rates (Tables 3,4). Multivariate analysis indicated that factors significantly associated with inpatient all-cause mortality were $\mathrm{AF}$ [hazard ratio (HR): $1.52,95 \%$ confidence interval (CI): $1.19-1.95, \mathrm{P}<0.001$ ], SOFA score (HR: $1.09,95 \%$ CI: $1.06-1.12, \mathrm{P}<0.001)$, WBC count (HR: $1.04,95 \%$ CI: 1.03-1.05, $\mathrm{P}<0.001$ ), platelet count (HR: $1.00,95 \% \mathrm{CI}$ : $0.99-1.00, \mathrm{P}<0.001$ ), and INR (HR: $1.30,95 \%$ CI: $1.17-$ 1.46, $\mathrm{P}<0.001)$ (Table 3). Kaplan-Meier analysis and Cox regression estimated survival analysis (both showed that patients with $\mathrm{AF}$ had a higher inpatient all-cause mortality rate than patients without $\mathrm{AF}(\mathrm{P}=0.024$ and $\mathrm{P}=0.025$, respectively) (Figure 2A,2B).

As shown in Table 4, multivariate analysis indicated that variables significantly associated with 4-year all-cause mortality were age (HR: 1.01, 95\% CI: 1.00-1.02, $\mathrm{P}=0.010$ ), AF (HR: 1.55, 95\% CI: 1.12-2.13, $\mathrm{P}=0.008)$, SOFA score (HR: 1.05 , 95\% CI: 1.01-1.09, P=0.005), MELD score (HR: 1.01, 95\% CI: 1.01-1.02, $\mathrm{P}<0.001$ ), RDW (HR: 1.06, 95\% CI: 1.02-1.11, $\mathrm{P}=0.002$ ), WBC count (HR: 1.02, 95\% CI: $1.00-1.04, \mathrm{P}=0.014$ ), albumin level (HR: $0.76,95 \% \mathrm{CI}$ : $0.62-0.92, \mathrm{P}=0.006)$, and platelet count (HR: $1.00,95 \% \mathrm{CI}$ : $1.00-1.00, \mathrm{P}=0.001)$.

Kaplan-Meier analysis and Cox regression estimated survival analysis both showed that patients with AF had a higher 4-year all-cause mortality rate than patients without $\mathrm{AF}$ (both $\mathrm{P}<0.001$ ) (Figure 2C,2D).

Table 1 Characteristics of all patient inclusion (survivors and non-survivors)

\begin{tabular}{|c|c|c|c|c|}
\hline Parameter & All patients $(n=1,481)$ & Survivors $(n=1,087)$ & Non-survivors $(n=394)$ & $P$ value \\
\hline Sex & & & & 0.747 \\
\hline Male & $1,002(67.66)$ & $738(67.89)$ & $264(67.01)$ & \\
\hline Female & 479 (32.34) & $349(32.11)$ & $130(32.99)$ & \\
\hline Smoking history & $80(5.40)$ & $65(5.98)$ & $15(3.81)$ & 0.102 \\
\hline Ethnicity & & & & 0.002 \\
\hline White & 1,039 (70.20) & $770(70.90)$ & $269(68.27)$ & \\
\hline Black & $116(7.84)$ & $89(8.20)$ & $27(6.85)$ & \\
\hline
\end{tabular}

Table 1 (continued) 
Table 1 (continued)

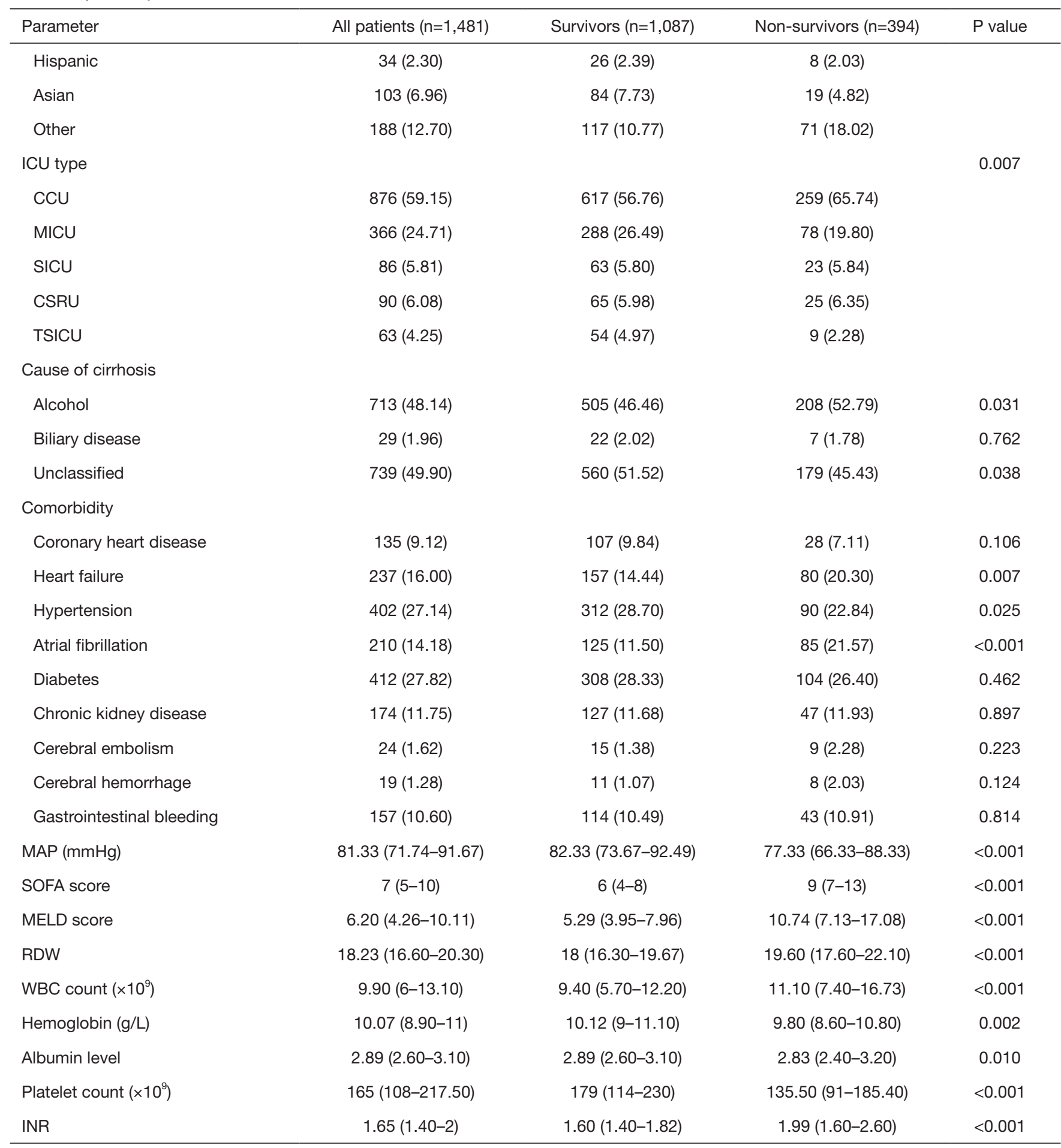

Data presented as mean \pm standard deviation, count (percentage), or median [interquartile range (IQR)]. Non-survivor, died during hospitalization. BMI, body mass index; CCU, cardiovascular care unit; CSRU, cardiac surgery intensive care unit; ICU, intensive care unit; INR, international normalized ratio; MAP, mean arterial pressure; MELD, model for end-stage liver disease; MICU, medical intensive care unit; RDW, red cell volume distribution width; SICU, surgery intensive care unit; SOFA, sequential organ failure assessment; TSICU, trauma surgery intensive care unit; WBC, white blood cell. 
Table 2 Unadjusted outcomes of patients with or without atrial fibrillation (AF)

\begin{tabular}{lccc}
\hline Parameter & No AF $(\mathrm{n}=1,271)$ & AF $(\mathrm{n}=210)$ & $\mathrm{P}$ value \\
\hline ICU LOS & $2.95(1.85-5.74)$ & $4.74(2.41-10.73)$ & $<0.001$ \\
Hospital LOS & $11.71(6.04-14.71)$ & $13.11(7.80-20.83)$ & $<0.001$ \\
Inpatient mortality & $309(24.31)$ & $85(40.48)$ & $<0.001$ \\
Cause of cirrhosis & & & \\
Alcohol & $625(49.17)$ & $6(41.90)$ & 0.051 \\
Biliary disease & $23(1.81)$ & $116(55.24)$ & 0.286 \\
Unclassified & $623(49.02)$ & $7(5-10)$ & 0.095 \\
SOFA score & $7(4-10)$ & $6.50(4.72-10.69)$ & 0.806 \\
MELD score & $6.10(4.16-10.01)$ & & 0.027 \\
\hline
\end{tabular}

Data reported as median (interquartile range) or count (percentage). ICU, intensive care unit; LOS, length of stay; MELD, model for endstage liver disease; SOFA, sequential organ failure assessment.

Table 3 Univariate and multivariate Cox regression analysis results of independent variables and inpatient all-cause mortality

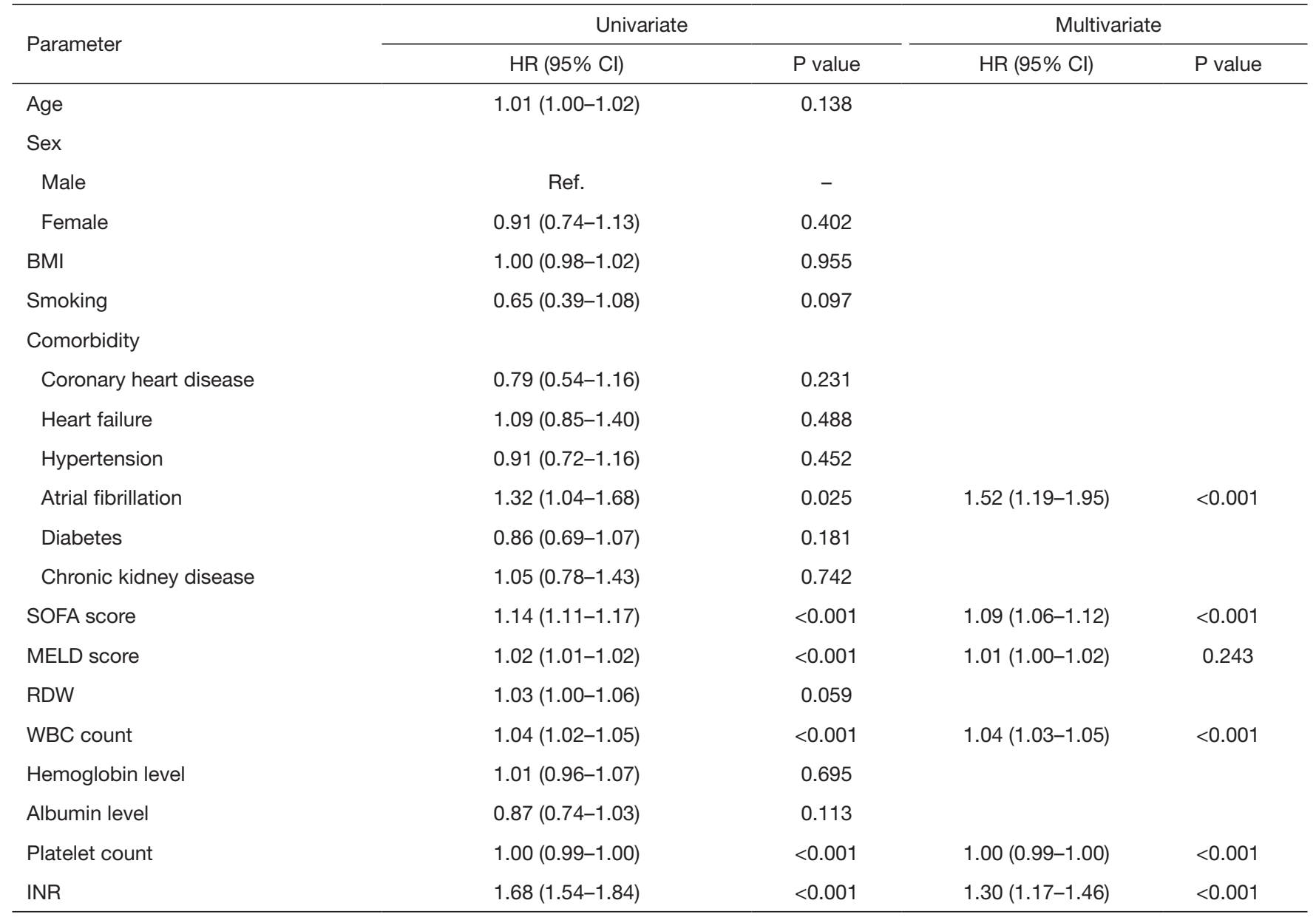

Variables significant in the univariate analysis $(\mathrm{P}<0.05)$ were entered into the multivariate model. Variables with a value of $\mathrm{P}<0.05$ in both the univariate and multivariate analyses were considered factors associated with inpatient mortality. $\mathrm{BMI}$, body mass index; $\mathrm{Cl}$, confidence interval; HR, hazard ratio; INR, international normalized ratio; MELD, model for end-stage liver disease; RDW, red cell volume distribution width; SOFA, sequential organ failure assessment; WBC, white blood cell. 

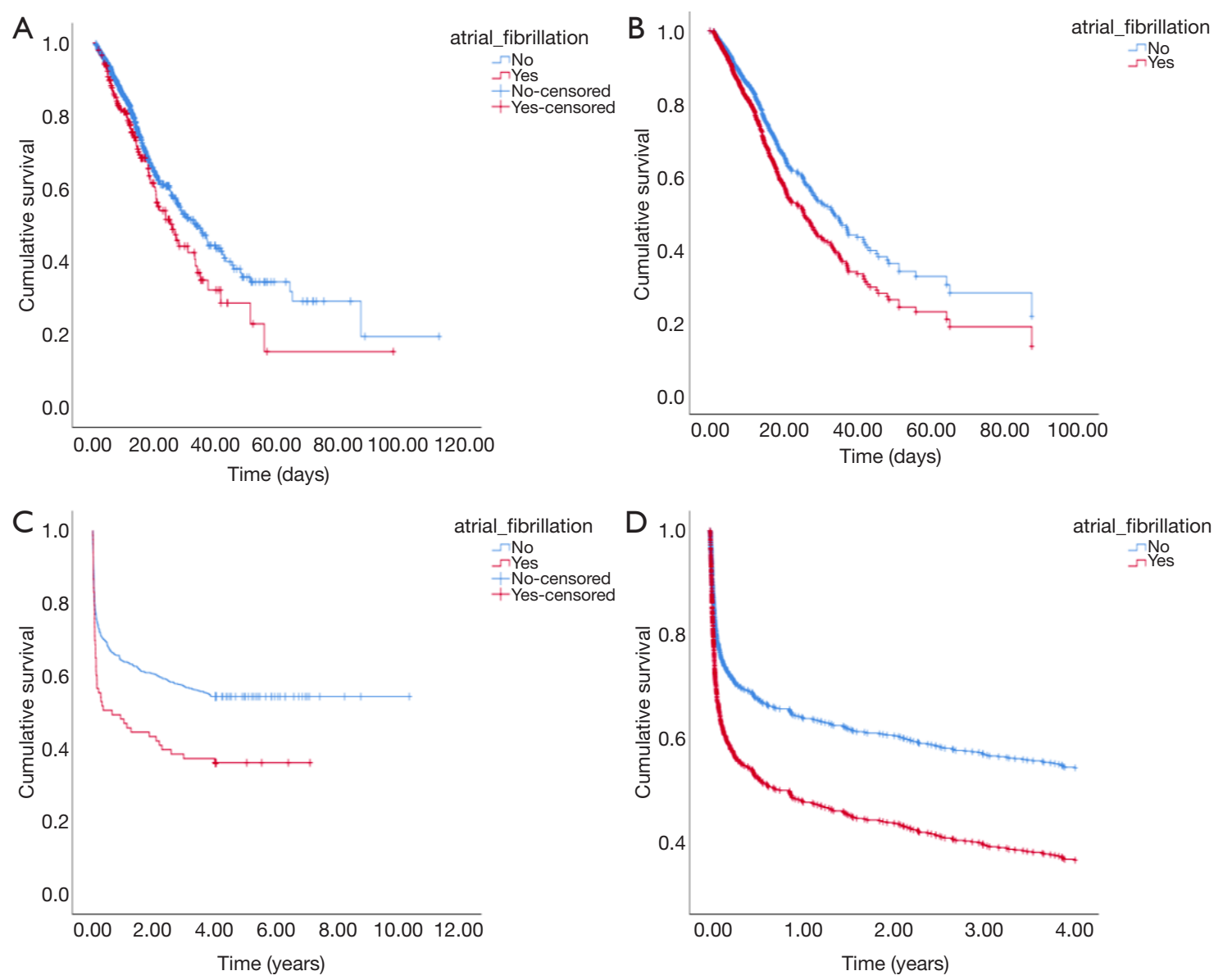

Figure 2 Survival curve analysis of AF in liver cirrhosis patient. Kaplan-Meier plots of inpatient Cumulative survival (A). Cox regression plots of inpatient Cumulative survival (B). Kaplan-Meier plots of 4-year Cumulative survival (C). Cox regression plots of 4-year Cumulative survival (D).

\section{Association between anticoagulation (AC) and study outcomes}

Unadjusted outcomes of cirrhosis patient with AF who received AC or not were summarized in Table 5. There were 57 patients received AC (27.14\%), with 56 received warfarin and one received Direct-acting oral anticoagulants (DOACs). The rates of cerebral embolism, cerebral hemorrhage and gastrointestinal bleeding were similar between the AC and non-AC groups. The inpatient allcause mortality rate was significantly lower in patients with AC than those without AC $(8.77 \%$ vs. $52.29 \%$, $\mathrm{P}<0.001)$. ICU length of stay was shorter in patients with AC $(\mathrm{P}=0.024)$, while total hospital length of stay was longer $(\mathrm{P}=0.009)$.

Kaplan-Meier analysis showed that patients with AC had a lower inpatient all-cause mortality rate than those without AC $(\mathrm{P}<0.001)$ (Figure 3A). Kaplan-Meier analysis for 4-year all-cause mortality showed that the survival curve crossed at about 2.5 years (Figure 3B). Landmark analysis showed that patients with $\mathrm{AC}$ had a lower all-cause mortality rate than those without AC before 2.5 years $(\mathrm{P}=0.012)$ (Figure 3C). Whereas the opposite finding was recorded for 2.5 years later follow-up. But this difference was not statistical significance $(\mathrm{P}=0.153)$ (Figure $3 D)$.

\section{Discussion}

The findings of the present study indicated that the overall prevalence of AF in ICU patients with liver cirrhosis was approximately $14.18 \%$, and importantly, the presence of $\mathrm{AF}$ in patients with liver cirrhosis hospitalized in an ICU was an independent predictor of increased risk of inpatient and 4-year all-cause mortality. Patients with $\mathrm{AF}$ also had a significantly longer ICU length of stay and hospital length 
Table 4 Univariate and multivariate Cox regression results of independent variables and 4-year all-cause mortality

\begin{tabular}{|c|c|c|c|c|}
\hline Parameters & \multicolumn{2}{|c|}{ Univariate } & \multicolumn{2}{|c|}{ Multivariate } \\
\hline Age & $1.01(1.00-1.02)$ & 0.031 & $1.01(1.00-1.02)$ & 0.010 \\
\hline \multicolumn{5}{|l|}{ Sex } \\
\hline Male & ref. & - & & \\
\hline BMI & $1.00(0.95-1.05)$ & 0.911 & & \\
\hline Smoking & $0.83(0.31-2.21)$ & 0.704 & & \\
\hline \multicolumn{5}{|l|}{ Comorbidity } \\
\hline Coronary heart disease & $1.04(0.71-1.52)$ & 0.850 & & \\
\hline Atrial fibrillation & $1.65(1.23-2.21)$ & $<0.001$ & $1.55(1.12-2.13)$ & 0.008 \\
\hline Diabetes & $0.96(0.76-1.23)$ & 0.757 & & \\
\hline Chronic kidney disease & $0.63(0.38-1.05)$ & 0.074 & & \\
\hline SOFA score & $1.10(1.07-1.13)$ & $<0.001$ & $1.05(1.01-1.09)$ & 0.005 \\
\hline MELD score & 1.02 (1.02-1.03) & $<0.001$ & $1.01(1.01-1.02)$ & $<0.001$ \\
\hline RDW & $1.12(1.08-1.16)$ & $<0.001$ & $1.06(1.02-1.11)$ & 0.002 \\
\hline WBC count & $1.02(1.00-1.03)$ & 0.025 & $1.02(1.00-1.04)$ & 0.014 \\
\hline Hemoglobin level & $0.92(0.86-0.97)$ & 0.005 & $0.95(0.89-1.01)$ & 0.121 \\
\hline
\end{tabular}

Variables significant in the univariate analysis $(\mathrm{P}<0.05)$ were entered into the multivariate model. Variables with a value of $\mathrm{P}<0.05$ in both the univariate and multivariate analyses were considered factors associated with 4-year mortality. BMI, body mass index; $\mathrm{Cl}$, confidence interval; HR, hazard ratio; INR, international normalized ratio; MELD, model for end-stage liver disease; RDW, red cell volume distribution width; SOFA, sequential organ failure assessment; WBC, white blood cell.

Table 5 Unadjusted outcomes of cirrhosis patient with atrial fibrillation (AF) who received anticoagulation (AC) or not

\begin{tabular}{|c|c|c|c|c|}
\hline Parameter & All patients $(n=210)$ & $A C(n=57)$ & Non-AC ( $n=153)$ & $P$ value \\
\hline Hospital LOS & $16.3(7.83-20.75)$ & $19.25(11.08-22.88)$ & $15.19(6.08-19.38)$ & 0.009 \\
\hline Cerebral embolism & $3(1.43)$ & 0 & $3(0.02)$ & 0.287 \\
\hline Cerebral hemorrhage & $3(1.43)$ & $3(5.26)$ & 0 & 0.218 \\
\hline Inpatient mortality & $85(40.48)$ & $5(8.77)$ & 80 (52.29) & $<0.001$ \\
\hline
\end{tabular}

Data reported as median (interquartile range) or count (percentage). ICU, intensive care unit; LOS, length of stay. 

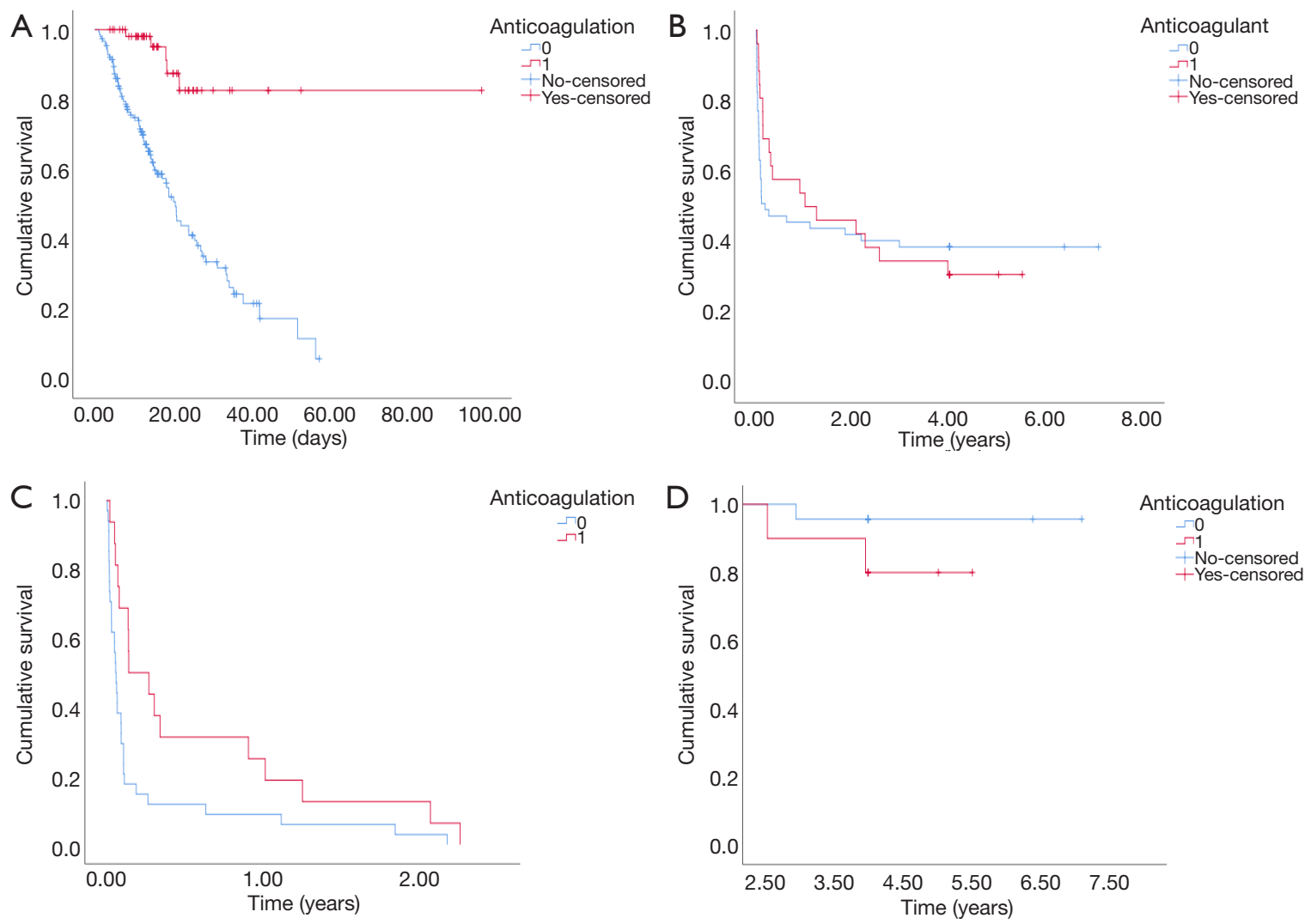

Figure 3 Kaplan-Meier survival curve analysis of AC in liver cirrhosis patients with AF. Kaplan-Meier plots of inpatient cumulative survival (A). Kaplan-Meier plots of 4-year cumulative survival (B). Kaplan-Meier plots of Landmark analysis before 2.5 years cumulative survival (C). Kaplan-Meier plots of Landmark analysis after 2.5 years cumulative survival (D).

of stay and a significantly higher MELD score than those without $\mathrm{AF}$.

Previously published studies examining whether or not liver cirrhosis is associated with an increased rate of $\mathrm{AF}$ have provided inconsistent results. A study in 2012 of patients with liver cirrhosis found that only $0.15 \%$ had $\mathrm{AF}$, a percentage much lower than that seen in the general population (20). A study published in 2016 of 335 patients with liver cirrhosis who were followed up for 24 months found that liver cirrhosis was not a predisposing factor for $\mathrm{AF}$ (12).

More recent studies, however, have suggested that liver cirrhosis and other gastrointestinal and liver diseases are risk factors for $\mathrm{AF}(4,9)$. Lee et al. performed a nationwide, population-based study and reported that, during the 9-year follow up, the rate of new-onset $\mathrm{AF}$ in patients with cirrhosis was $3.1 \%$, which was much higher than that of patients without cirrhosis (HR: 1.46) (10). A 2018 retrospective data analysis reported that the incidence of $\mathrm{AF}$ was significantly higher in patients with liver disease than those without, and that a higher MELD score was predictive of new-onset AF (11). A recent meta-analysis by Chokesuwattanaskul et al. found that the overall estimated prevalence of AF in patients with cirrhosis was 5\% (14), which is higher than that in the general population reported by Hindricks et al. (6). Another recent meta-analysis also found that non-alcoholic fatty liver disease was a predictor of $\mathrm{AF}$ (8). Liver cirrhosis patients commonly had increased left atrium (LA) size, left ventricular hypertrophy (21), and prolonged QTc interval (22). These are regarded as the main mechanisms for the increased incidence of $\mathrm{AF}$ in patients with liver cirrhosis (8). Our results indicated that the prevalence of $\mathrm{AF}$ in patients with liver cirrhosis was approximately $14 \%$, which is markedly higher than that reported in the aforementioned studies $(6,14)$. The higher rate is likely because our study only included critically ill patients with cirrhosis.

Studies on the impact of AF on the mortality of patients with cirrhosis have also provided inconsistent results. 
In their studies, Lee et al. and Mwalitsa et al. found no significant correlation between $\mathrm{AF}$ and mortality in patients with cirrhosis $(10,12)$. However, a study by Darrat et al. published in 2020 found that hospitalized patients with cirrhosis with AF had a significantly higher inpatient mortality rate than those without AF (13). A 2019 metaanalysis also demonstrated a statistically significant increased mortality risk in cirrhotic patients with AF (14). Vannucci et al. reported that, in patients undergoing liver transplantation, 30-day and 1-year survival rates were lower in patients with a preoperative diagnosis of $\mathrm{AF}$ (23). There are few published studies on the effect of AF on mortality in patients with liver cirrhosis. Our findings are consistent with those of the aforementioned studies (13) in that AF was found to be an independent predictor of in-patient and 4-year all-cause mortality. Furthermore, our study included 1,481 patients, and approximately half were followed up for more than 4 years.

In the subgroup analysis, we studied the safety and benefits of the use of $\mathrm{AC}$ in patients with cirrhosis and $\mathrm{AF}$. The bleeding risk of patients with liver cirrhosis is higher than that of the normal population, and we are more cautious in choosing whether to give AC therapy. A retrospective longitudinal study showed that warfarin and DOACs were associated with reduced all-cause mortality. Warfarin was associated with more bleeding compared to no AC. DOACs had a lower incidence of bleeding compared to warfarin in exploratory analyses (24). Our study showed that the use of $\mathrm{AC}$ is relatively safe. The risk of cerebral hemorrhage and gastrointestinal hemorrhage was not statistically different between the two groups. And AC can reduce the mortality rate within 2.5 years. As few cases in subgroup analysis, and just one patient us DOACs, future studies should prospectively investigate these observed associations.

The present study had limitations that should be considered. This was a retrospective database cohort study, the results need to be further verified in a prospective study. Limitations of the database used also imposed limitations on the study. For example, data, such as the type of AF and treatment medications, were not available in the database. In addition, cause of death was not specific in the database, and therefore, we could not examine disease-specific mortality.

\section{Conclusions}

Critically ill patients with liver cirrhosis have a high rate of $\mathrm{AF}$, and $\mathrm{AF}$ is an independent risk for inpatient and 4-year all-cause mortality.

\section{Acknowledgments}

Funding: This work was supported by the Guangzhou Science and Technology Project (grant number: 201802010048), the National Natural Science Foundation of China (grant number: 81773176), the National Natural Science Foundation of China (grant number: 81900320), the Medical Science and Technology Foundation of Guangdong Province (grant number: C2019107), the Basic Research Funding of Sun Yat-Sen University (grant number: 19ykpy40), the Natural Science Foundation of Guangdong Province (grant number: 2020A1515010599), and the Natural Science Foundation of Guangdong Province (grant number: 2018A0303130070).

\section{Footnote}

Reporting Checklist: The authors have completed the STROBE reporting checklist. Available at https://dx.doi. org/10.21037/atm-21-3111

Conflicts of Interest: All authors have completed the ICMJE uniform disclosure form (available at https://dx.doi. org/10.21037/atm-21-3111). The authors have no conflicts of interest to declare.

Ethical Statement: The authors are accountable for all aspects of the work in ensuring that questions related to the accuracy or integrity of any part of the work are appropriately investigated and resolved. The present study was approved by the Institutional Review Boards of Massachusetts Institute of Technology (Cambridge, MA, USA) and Beth Israel Deaconess Medical Center (Boston, MA, USA) (reference number: 32540900). Because of the retrospective nature of the study, the requirement of patient informed consent was waived. The study was conducted in accordance with the Declaration of Helsinki (as revised in 2013).

Open Access Statement: This is an Open Access article distributed in accordance with the Creative Commons Attribution-NonCommercial-NoDerivs 4.0 International License (CC BY-NC-ND 4.0), which permits the noncommercial replication and distribution of the article with the strict proviso that no changes or edits are made and the original work is properly cited (including links to both the formal publication through the relevant DOI and the license). See: https://creativecommons.org/licenses/by-nc$\mathrm{nd} / 4.0 \%$. 


\section{References}

1. Zhou WC, Zhang QB, Qiao L. Pathogenesis of liver cirrhosis. World J Gastroenterol 2014;20:7312-24.

2. Carvalho MVH, Kroll PC, Kroll RTM, et al. Cirrhotic cardiomyopathy: the liver affects the heart. Braz J Med Biol Res 2019;52:e7809.

3. Cao Y, Ji C, Lu L. Mesenchymal stem cell therapy for liver fibrosis/cirrhosis. Ann Transl Med 2020;8:562.

4. Long MT, Ko D, Arnold LM, et al. Gastrointestinal and liver diseases and atrial fibrillation: a review of the literature. Therap Adv Gastroenterol 2019;12:1756284819832237.

5. Benjamin EJ, Muntner P, Alonso A, et al. Heart Disease and Stroke Statistics-2019 Update: A Report From the American Heart Association. Circulation 2019;139:e56-e528.

6. Hindricks G, Potpara T, Dagres N, et al. 2020 ESC Guidelines for the diagnosis and management of atrial fibrillation developed in collaboration with the European Association for Cardio-Thoracic Surgery (EACTS): The Task Force for the diagnosis and management of atrial fibrillation of the European Society of Cardiology (ESC) Developed with the special contribution of the European Heart Rhythm Association (EHRA) of the ESC. Eur Heart J 2021;42:373-498.

7. Kang MK, Park JG, Kim MC. Association between atrial fibrillation and advanced liver fibrosis in patients with nonalcoholic fatty liver disease. Yonsei Med J 2020;61:860-7.

8. Zhou Y, Lai C, Peng C, et al. Nonalcoholic fatty liver disease as a predictor of atrial fibrillation: a systematic review and meta-analysis. Postepy Kardiol Interwencyjnej 2017;13:250-7.

9. Mozos I. Arrhythmia risk in liver cirrhosis. World J Hepatol 2015;7:662-72.

10. Lee H, Choi EK, Rhee TM, et al. Cirrhosis is a risk factor for atrial fibrillation: A nationwide, population-based study. Liver Int 2017;37:1660-7.

11. Huang WA, Dunipace EA, Sorg JM, et al. Liver disease as a predictor of new-onset atrial fibrillation. J Am Heart Assoc 2018;7:e008703.

12. Mwalitsa JP, Maimone S, Filomia R, et al. Atrial fibrillation in patients with cirrhosis. Liver Int 2016;36:395-400.

13. Darrat YH, Smer A, Elayi CS, et al. Mortality and morbidity in patients with atrial fibrillation and liver cirrhosis. World J Cardiol 2020;12:342-50.

14. Chokesuwattanaskul R, Thongprayoon C, Bathini T, et al. Epidemiology of atrial fibrillation in patients with cirrhosis and clinical significance: a meta-analysis. Eur J
Gastroenterol Hepatol 2019;31:514-9.

15. Saeed M, Villarroel M, Reisner AT, et al. Multiparameter intelligent monitoring in intensive care II (MIMIC-II): a public-access intensive care unit database. Crit Care Med 2011;39:952-60.

16. Johnson AEW, Pollard TJ, Shen L, et al. MIMICIII, a freely accessible critical care database. Sci Data 2016;3:160035.

17. Kamath PS, Wiesner RH, Malinchoc M, et al. A model to predict survival in patients with end-stage liver disease. Hepatology 2001;33:464-70.

18. Vincent JL, de Mendonça A, Cantraine F, et al. Use of the SOFA score to assess the incidence of organ dysfunction/ failure in intensive care units: results of a multicenter, prospective study. Working group on "sepsis-related problems" of the European Society of Intensive Care Medicine. Crit Care Med 1998;26:1793-800.

19. Maeng M, Tilsted HH, Jensen LO, et al. Differential clinical outcomes after 1 year versus 5 years in a randomised comparison of zotarolimus-eluting and sirolimus-eluting coronary stents (the SORT OUT III study): a multicentre, open-label, randomised superiority trial. Lancet 2014;383:2047-56.

20. Zamirian M, Sarmadi T, Aghasadeghi K, et al. Liver cirrhosis prevents atrial fibrillation: A reality or just an illusion? J Cardiovasc Dis Res 2012;3:109-12.

21. Pozzi M, Carugo S, Boari G, et al. Evidence of functional and structural cardiac abnormalities in cirrhotic patients with and without ascites. Hepatology 1997; 26:1131-7.

22. Bernardi M, Maggioli C, Dibra V, et al. QT interval prolongation in liver cirrhosis: innocent bystander or serious threat? Expert Rev Gastroenterol Hepatol 2012;6:57-66.

23. Vannucci A, Rathor R, Vachharajani N, et al. Atrial fibrillation in patients undergoing liver transplantation-a single-center experience. Transplant Proc 2014;46:1432-7.

24. Serper M, Weinberg EM, Cohen JB, et al. Mortality and hepatic decompensation in patients with cirrhosis and atrial fibrillation treated with anticoagulation. Hepatology 2021;73:219-32.

(English Language Editor: R. Scott)

Cite this article as: Luo Y, Wu B, Wu Y, Peng L, Li Z, Zhu J, Su Z, Liu J, Li S, Chong Y. Atrial fibrillation increases inpatient and 4-year all-cause mortality in critically ill patients with liver cirrhosis. Ann Transl Med 2021;9(15):1239. doi: 10.21037/atm21-3111 